\title{
Malignant Pyriform Fossa Neoplasm
}

National Cancer Institute

\section{Source}

National Cancer Institute. Malignant Pyriform Fossa Neoplasm. NCI Thesaurus. Code C3531.

A primary or metastatic malignant neoplasm that affects the pyriform sinus. 